\title{
Erratum to: Electing a Proportional All-Party, Power-Sharing Executive
}

Erratum to: P. Emerson, From Majority Rule to Inclusive Politics, DOI 10.1007/978-3-319-23500-4_6

The caption of Table 6.22 has been incorrectly published as "Two votes from Party L" on Page 95 and in the list of tables on page xxvi. The correct Table caption is "The quotas gained and MBC sums awarded"

The online version of the original chapter can be found under DOI 10.1007/978-3-319-23500-4_6 